\section{International Scientific Journal Theoretical \& Applied Science}

\author{
p-ISSN: 2308-4944 (print) e-ISSN: 2409-0085 (online) \\ Year: $2015 \quad$ Issue: 09 Volume: 29
}

Published: $30.09 .2015 \quad$ http://T-Science.org
Nikolai Vladimirovich Semenchenko

Student of the Department «Equipment and technology of machine-building production»" FSBEI HPE «Togliatti State University», Russia bonder64@mail.ru

Konstantin Olegovich Hryachkov Student of the Department «Equipment and technology of machine-building production» FSBEI HPE «Togliatti State University», Russia

SECTION 7. Mechanics and machine construction.

\title{
TRENDS OF THE DEFORMATION CLADDING BY FLEXIBLE TOOL
}

Abstract: This report describes the basic trends of the deformation cladding by flexible tool, changes have occurred during development of this method. Main directions during development of the deformation cladding had been classified. The existing schemes of deformation cladding, the application of additional effects, constructions of alloy elements, constructions of flexible tools have been analyzed. We discussed their strengths and weaknesses. Possible direction of further development was revealed.

Key words: flexible tool; cladding; coating method; scheme of the process; constructions of alloy elements; additional effects; constructions of flexible tools.

Language: Russian

Citation: Semenchenko NV, Hryachkov KO (2015) TRENDS OF THE DEFORMATION CLADDING BY FLEXIBLE TOOL. ISJ Theoretical \& Applied Science 09 (29): 105-114.

Soi: http://s-o-i.org/1.1/TAS-09-29-21 Doi: crossef http://dx.doi.org/10.15863/TAS.2015.09.29.21

\section{ТЕНДЕНЦИИ РАЗВИТИЯ МЕТОДА ДЕФОРМАЦИОННОГО ПЛАКИРОВАНИЯ ГИБКИМ ИНСТРУМЕНТОМ}

Аннотация: В данной статье рассмотрены основные тенденции развития метода деформационного плакирования гибким инструментом, рассмотрены изменения, произошедиие с данным методом. Классифицированы основные направления развития процесса ДПГИ, проанализированы предложенные схемы реализации прочесса, применение дополнительных физических воздействий, конструкции элемента материала покрытия и конструкиии гибкого инструмента; рассмотрены их слабые и сильные стороны. Выявлено возможное направление дальнейшего развития данного метода нанесения покрытий.

Ключевые слова: гибкий инструмент; плакирование; метод нанесения покрытий; схема реализации процесса; конструкиии ЭМП; дополнительные воздействия; конструкции гибкого инструмента.

Деформационное плакирование - один из универсальных методов модификации поверхностного слоя рабочих поверхностей деталей, позволяющий добиться не только упрочнения поверхности, но и, одновременно, нанести на нее покрытие [1]. В отличие от других методов нанесения служебных покрытий, данный метод не требует предварительной обработки поверхности изделия, так как очистка поверхности осуществляется в процессе плакирования.

В настоящее время происходит внедрение данного метода в разные отрасли промышленности, в частности в производства биметаллической ленты и проволоки, нанесения служебных покрытий на детали, работающие в узлах трения, восстановления изношенных деталей, нанесения защитных и антикоррозионных покрытий. Метод деформационного плакирования с каждым годом претерпевает модернизацию и развитие. На рис. 1 представлены основные направления развития метода плакирования.

Представленная классификация показывает, что развитие плакирования происходит в разных направления, изменяются конструкции установок для нанесения покрытия, процесс плакирования дополняется физическими воздействиями с целью его интенсификации, совершенствуются составы наносимого материала, выбираются оптимальные скорости обработки, принципиальные схемы реализации процесса, улучшаются конструкции гибкого инструмента (механической щетки) и многое другое. 


\begin{tabular}{|c|c|c|c|c|c|c|}
\hline Impact Factor: & $\begin{array}{l}\text { ISRA (India) } \\
\text { ISI (Dubai, UAE } \\
\text { GIF (Australia) } \\
\text { JIF }\end{array}$ & $\begin{array}{l}=1.344 \\
=0.829 \\
=0.356 \\
=1.500\end{array}$ & $\begin{array}{l}\text { SIS (USA) } \\
\text { PИНЦ (Russia) } \\
\text { ESJI (KZ) } \\
\text { SJIF (Morocco) }\end{array}$ & $\begin{array}{l}=0.912 \\
=0.179 \\
=1.042 \\
=2.031\end{array}$ & ICV (Poland) & $=6.630$ \\
\hline
\end{tabular}

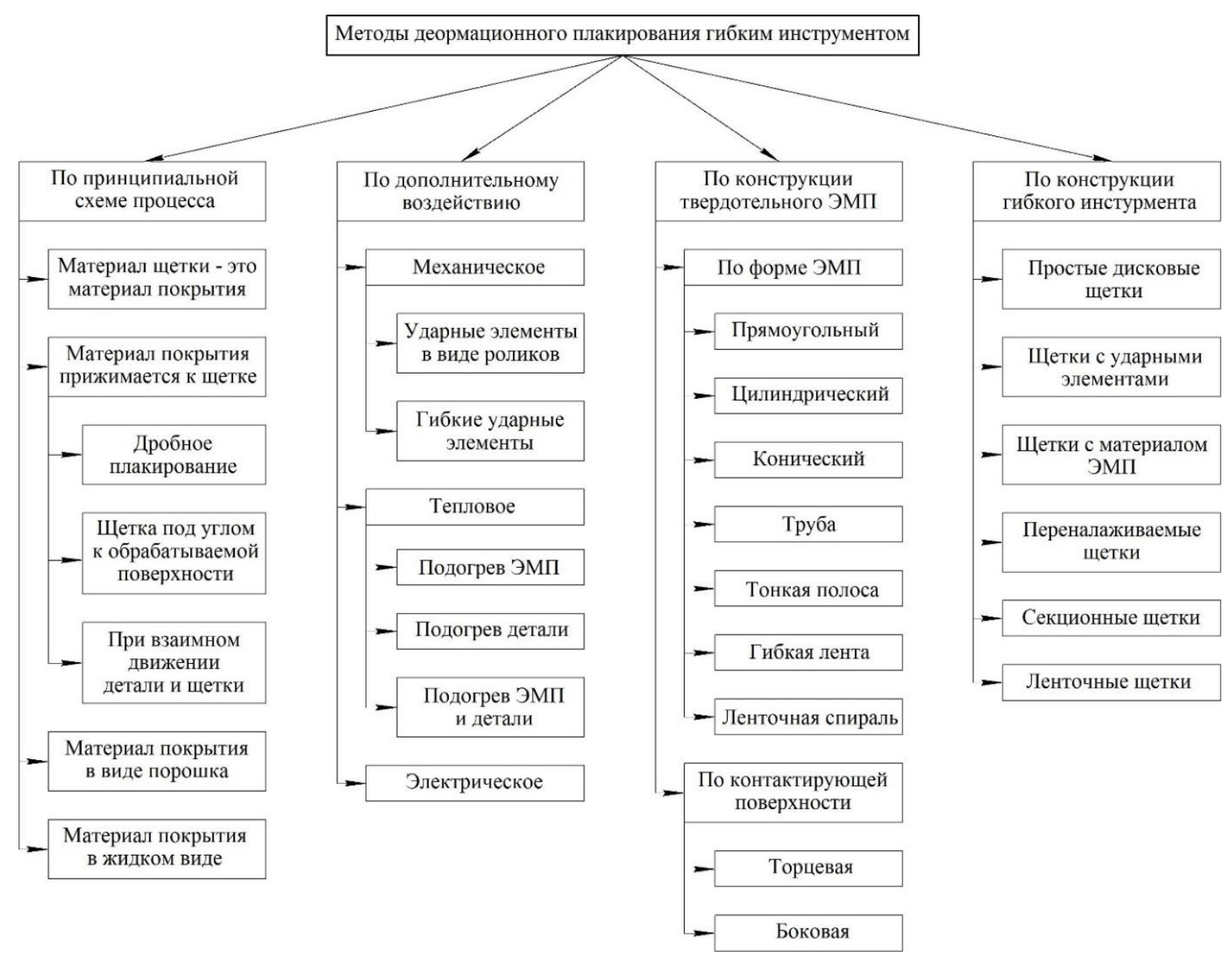

Рисунок 1 - Классификация методов ДПГИ.

\section{СХЕМЫ РЕАЛИЗАЦИИ ПРОЦЕССА ПЛАКИРОВАНИЯ}

Существую две принципиально различные схемы реализации процесса деформационного плакирования. Первая (рис. 2), предложенная в 1960 году изобретателями: Смирновым И.М.,
Николаевым Н.А., Крыловым С.Д. [2], отличается тем, что в качестве материала наносимого покрытия выступают проволочные элементы гибкого инструмента. Данную схему рекомендуется применять ее авторами для нанесения серебряного покрытия.

ISPC Modern research and development, 


\begin{tabular}{|c|c|c|c|c|c|c|}
\hline Impact Factor: & $\begin{array}{l}\text { ISRA (India) } \\
\text { ISI (Dubai, UAE } \\
\text { GIF (Australia) } \\
\text { JIF }\end{array}$ & $\begin{array}{l}=1.344 \\
=0.829 \\
=0.356 \\
=1.500\end{array}$ & $\begin{array}{l}\text { SIS (USA) } \\
\text { PИHЦ (Russia) } \\
\text { ESJI (KZ) } \\
\text { SJIF (Morocco) }\end{array}$ & $\begin{array}{l}=0.912 \\
=0.179 \\
=1.042 \\
=2.031\end{array}$ & ICV (Poland) & $=6.630$ \\
\hline
\end{tabular}

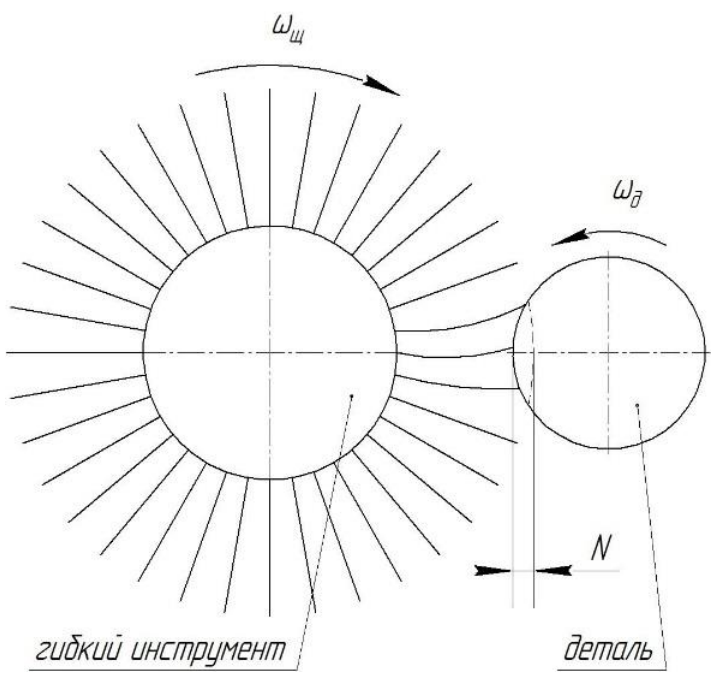

Рисунок 2 - Схема ДПГИ №1.

Она получила свое развитие в работе [3], где рекомендовано ее применение при нанесении никеля, черных и тугоплавких металлов.

Вторая же схема (рис. 3) предложена изобретателем Абиндером А.А. еще в 1937 году [4]. В которой механическая щетка играет роль механического переносчика частиц с элемента материала покрытия на обрабатываемую поверхность детали.
Именно данная схема плакирования приобрела большую популярность и получила свое развитие во многих научных работах. В таких как работы исследователей из Магнитогорского государственного технического университета, где была предложена схема дробного плакирования (рис. 4), разработанная с целью получения покрытий толщиной от 100 до 1000 мкм и более [5].

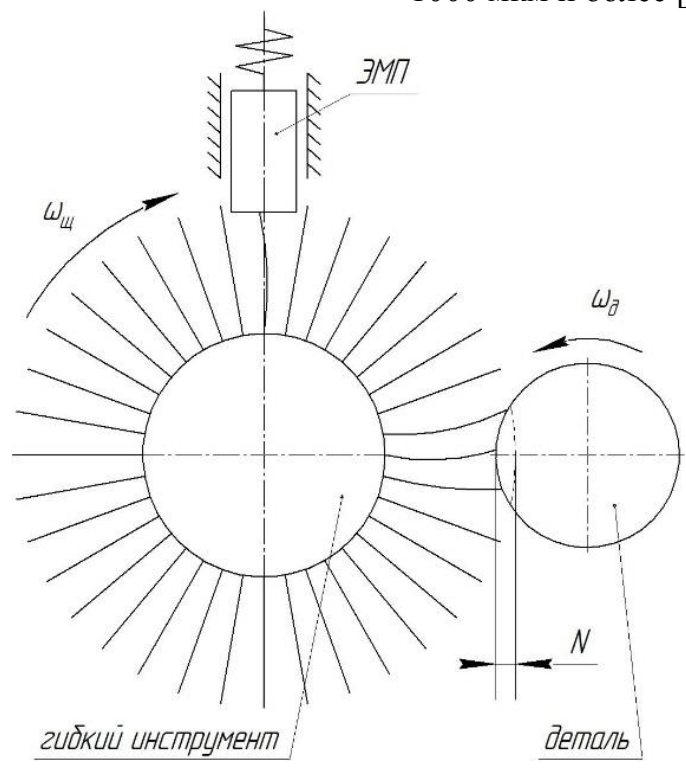

Рисунок 3 - Схема ДПГИ №2.

Отличие данной схемы состоит в сообщении дополнительного движения обрабатываемой детали в направлении перпендикулярной оси щетки, что позволяет достичь эффекта многократного временного взаимодействия гибких элементов инструмента и обрабатываемой поверхности детали. 


\begin{tabular}{|c|c|c|c|c|c|c|}
\hline Impact Factor: & $\begin{array}{l}\text { ISRA (India) } \\
\text { ISI (Dubai, UAE } \\
\text { GIF (Australia) } \\
\text { JIF }\end{array}$ & $\begin{array}{l}=1.344 \\
=0.829 \\
=0.356 \\
=1.500\end{array}$ & $\begin{array}{l}\text { SIS (USA) } \\
\text { PИHЦ (Russia) } \\
\text { ESJI (KZ) } \\
\text { SJIF (Morocco) }\end{array}$ & $\begin{array}{l}=0.912 \\
=0.179 \\
=1.042 \\
=2.031\end{array}$ & ICV (Poland) & $=6.630$ \\
\hline
\end{tabular}

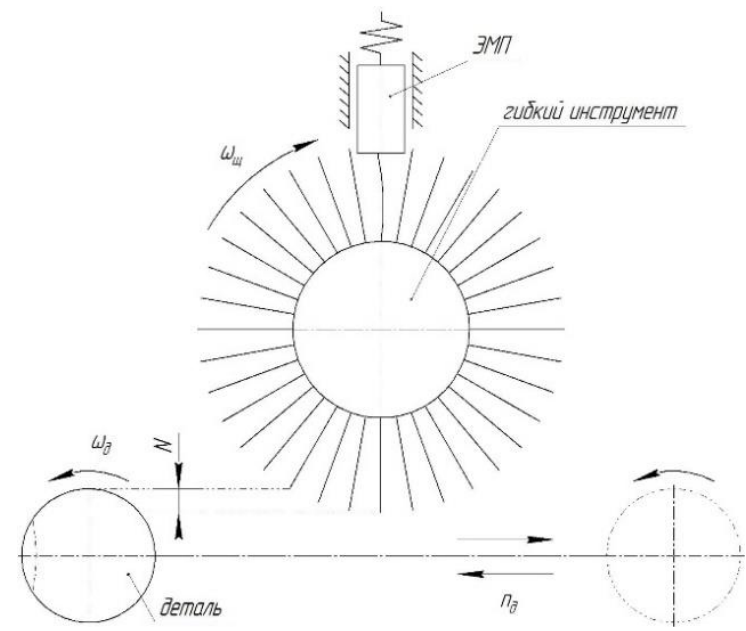

Рисунок. 4 - Схема дробного плакирования.

Так же механическая щетка может устанавливаться под углом к плакируемой поверхности детали (рис. 5), так при плакировании деталей цилиндрической формы, продольные оси обрабатываемой детали и щетки могут перекрещиваться в пространстве, то есть их проекции могут быть перпендикулярны, либо пересекаться под углом 25-45 градусов [4, 6-16].
Чаще всего данная схема применяется для плакирования проволоки или ленты, что снижает разнотолщинность наносимого на нее покрытия. Данный метод получил свое развитие в работе [17], где применена оригинальная траектория движения плакируемой ленты и в работе [18], где добавлено возмущающее воздействие в виде кручения на обрабатываемую проволоку.

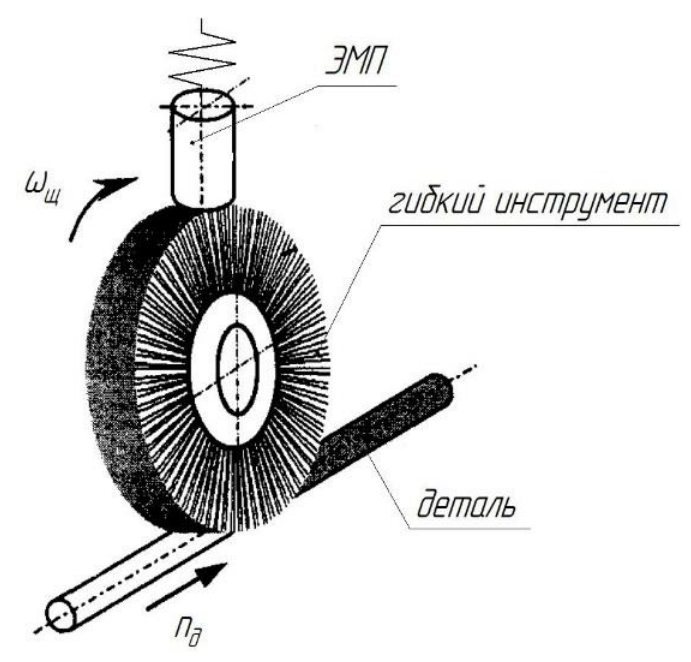

Рисунок 5 - Схема плакирования проволоки.

Еще одна схема реализации процесса плакирования представлена на рис. 6, которая чаще всего применяется при обработке плоских и широких деталей. В литературе $[1,19,20]$ обрабатываемая поверхность движется поступательно относительно щетки в двух направлениях, что позволяет наносить на поверхность покрытие с необходимым рисунком, например в виде сетки, для повышения маслоемкости поверхностного слоя. 


\begin{tabular}{|c|c|c|c|c|c|c|}
\hline Impact Factor: & $\begin{array}{l}\text { ISRA (India) } \\
\text { ISI (Dubai, UAE } \\
\text { GIF (Australia) } \\
\text { JIF }\end{array}$ & $\begin{array}{l}=1.344 \\
=0.829 \\
=0.356 \\
=1.500\end{array}$ & $\begin{array}{l}\text { SIS (USA) } \\
\text { PИHЦ (Russia) } \\
\text { ESJI (KZ) } \\
\text { SJIF (Morocco) }\end{array}$ & $\begin{array}{l}=0.912 \\
=0.179 \\
=1.042 \\
=2.031\end{array}$ & ICV (Poland) & $=6.630$ \\
\hline
\end{tabular}

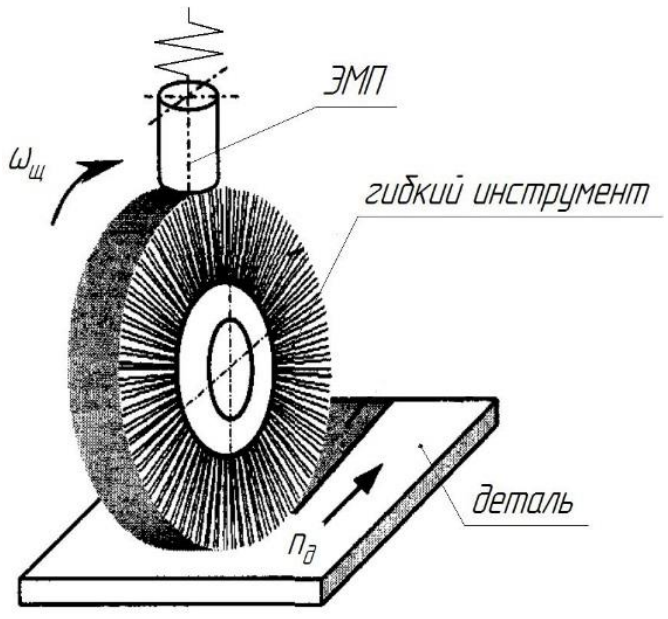

Рисунок 6 - Схема плакирования листа.

$\begin{array}{ccrr}\text { В процессе } & \text { развития, } & \text { метода } \\ \text { деформационного плакирования } & \text { гибким } \\ \text { инструментом } & \text { материал покрытия } & \text { стали } \\ \text { применять не только в виде } & \text { твердого бруска, } \\ \text { прижимаемого к к щетке. Изобретателями: }\end{array}$ Белевский Л.С., Харитонов А.О., Кутлубаев И.М., Серов Н.В. был предложен метод плакирования для нанесения тугоплавких материалов с использованием материала покрытия в виде порошка, подаваемого в непосредственно зону контакта гибкого инструмента с поверхность детали [21].

В работах же [22-26] изобретатели: Берсудский А.Л., Малышев В.П. и д.р. предлагают использовать материал покрытия в жидком состоянии на основе хлорида меди.

Bce данные подходы имеют место в производственной практике и показывают хорошие результаты, при правильном выборе метода для конкретных условий обработки.

\section{ПРИМЕНЕНИЕ ДОПОЛНИТЕЛЬНЫХ ВОЗДЕЙСТВИЙ}

Для интенсификации процесса деформационного плакирования в ряде работ авторы предлагают использовать дополнительное механическое, тепловое или электрическое воздействие на процесс.

Авторы изобретения [27] предлагают дополнительно воздействовать на обрабатываемую поверхность, пластически деформировать ее ударными элементами в виде роликов, установленных на гибком инструменте. Что повышает прочностные характеристики обработанной поверхности. В изобретении [28] для механического воздействия на поверхность предусмотрены гибкие ударные элементы, представляющие из себя жесткие ворсинки (жесткость которых больше жесткости остального ворса в 3-3000 раз), установленные в цилиндрической щетке в шахматном порядке, которые обеспечивают получение на поверхности обрабатываемого изделия рисунка определенного профиля. В работах $[29,30]$ используются интересные варианты комплектации ворса щеток, рабочие элементы которых состоят из ворсинок разной твердости и чередуются между собой. При этом гибкие элементы из более мягкого материала выполнены в виде трубок и армированы более твердым ворсом.

Другие же исследователи предлагают тепловое воздействие в виде предварительного нагрева плакируемой детали [31], что ускоряет начало процесса термомеханического переноса частиц материала покрытия на обрабатываемую поверхность. Так же для ускорения процесса переноса авторы работ [16, 32, 33] предлагают нагревать элемент из материала покрытия. Что и сделали изобретатели в работе [34], они нагревали элемент из материала покрытия пропусканием через него электрического тока, через специальный цилиндрический ролик. Применение данных методик позволяет осуществлять нанесение покрытий из твердых малопластичных металлов.

Электрическое же воздействие на процесс, представлено не только в работах $[34,35]$, где оно использовано для разогрева элемента из материала покрытия. Оно так же используется для расширения возможностей процесса плакирования путем нанесения более твердых, чем основа, токопроводящих материалов за счет совмещения процессов электрической эрозии и электроискрового легирования [36]. 


\section{ВАРИАНТЫ КОНСТРУКЦИЙ ТВЕРДОТЕЛЬНОГО ЭМП}

Одним из направлений развития технологии плакирования гибким инструментом является совершенствование конструкции, формы и размеров элемента материала покрытия. Был предложен целый ряд различных конструкций ЭМП в форме брусков прямоугольного сечения [37].

Изобретатели предлагали разные конструкции установок с цилиндрическими стержнями из материала покрытия [38-40] для получения однородных покрытий по всей длине обработки. Для экономии цветных металлов за счет сплавления с другими металлами и одновременного нанесения сплавов на поверхность авторы [41] снабдили устройство, помимо одного основного ЭМП, дополнительными брусками из различных металлов, причем бруски расположены в порядке возрастания температуры плавления материалов брусков, последовательно друг за другом по направлению вращения щетки. Предложены так же устройства для подачи элемента материала покрытия в зону обработки [42, 43], с помощью которых осуществляется контролируемый прижим ЭМП к торцевой поверхности механической щетки, где ЭМП устанавливается в направляющую трубку, а величина силы прижима контролируется натяжным винтом. Само же устройство устанавливается либо перпендикулярно к оси вращения гибкого инструмента [42], либо под специально рассчитанным углом к касательной, проведенной в точку касания бруска и щетки [43].

Авторы изобретения [34], с целью повышения качества покрытия, предлагают конец бруска, контактирующий с ворсом щетки, выполнить в виде конуса и установить его с натягом к щетке с возможностью вращения. Так же выполняют элементы материала покрытия в виде тонких полос [44] и гибких лент [35] для обработки, чаще всего, длинных металлических полос. Данные методы позволяют повысить равномерность и толщину наносимого покрытия, что положительно сказывается на качестве обработанных изделий.

С целью расширения технологических возможностей метода деформационного плакирования, за счет устранения эффекта перемешивания поверхностного слоя материала покрытия в зоне контакта с ворсом проволочного инструмента, ЭМП выполнен в виде полого цилиндра (трубы) [45]. Данный способ получил свое развитие в работе [46], где предложено использовать элемент из материала покрытия в виде набора концентрично расположенных цилиндров, изготовленных из различных материалов и расположенных в определенной последовательности начиная с наружного.

Для нанесения покрытий из малопластичных твердых материалов авторами работы [47] предложено изготавливать ЭМП в виде ленточной спирали, один конец которой установлен с возможностью контакта с механической щеткой, а ось спирали параллельна оси гибкого инструмента. Сама же спираль устанавливается в специальную теплоизолирующую оправку с направляющими.

Кроме применения более новых форм ЭМП в процессе развития были предложены и разные способы контакта элемента с периферией гибкого инструмента. Контакт торцевой поверхностью [38-43, 45, 46] можно считать «классическим», так как он предлагается к использованию начиная с самых ранних работ и используется чаще всего. Кроме него для экономии материала покрытия и снижения энергозатрат в процессе съема материала покрытия металлической щеткой и повышения стойкости гибкого инструмента, материал покрытия прижимается к щетке боковой поверхностью, а сам он имеет возможность осевого вращения в процессе обработки [48].

В развитие данного направления авторами [49] было предложено специальное устройство для подачи ЭМП в зону покрытия, выполненного в виде ролика и установленного на оси с возможностью вращения; снабженное механизмом прижима его посредством пружины к периферии щетки. Данное устройство отличается тем, что снабжено механизмом регулирования силы прижатия и тормозной колодкой, что дает возможность более гибкой настройки.

\section{ВАРИАНТЫ КОНСТРУКЦИЙ ГИБКОГО ИНСТРУМЕНТА}

В наши дни известно довольно много различных видов гибких инструментов, таких как: дисковые, валковые, кольцевые, торцевые, концевые, плоские, пучковые, секционные, ленточные щетки и даже иглофрезы [50]. Однако не все виды данных инструментов могут быть применены для деформационного плакирования, потому что при плакировании необходимо обеспечить не только определенное напряженнодеформированное состояние поверхностного слоя, но также и определенную тепловое воздействие [51, 52].

Чаще всего при нанесении покрытий методом плакирования используются простые и универсальные дисковые щетки, изготовленные из проволоки различных марок стали: 


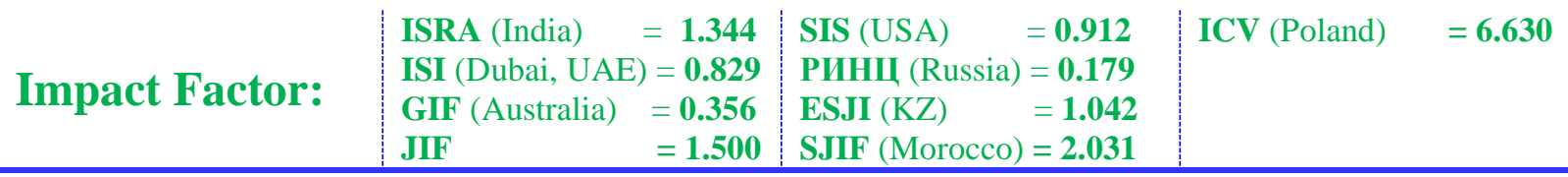

- легированной пружинной, термически обработанной (ГОСТ 14963-78),

- низкоуглеродистой, обработанной (ГОСТ 3282-74),

- углеродистой инструментальной У7А, У8А (ГОСТ 1435-99),

- пряди стальных канатов (ГОСТ 7372-79),

- металлокорд (ГОСТ 14311-85).

Не существует единого мнения о материале для производства ворса гибкого инструмента.

Для повышения качества обработки и расширения возможностей ДПГИ в конструкцию щеток вносятся различные дополнения. В работе [27] авторами, для достижения дополнительной пластической деформации, ворс щетки предлагается комбинировать с ударными элементами в форме роликов, которые в процессе обработки ударяются об обрабатываемую поверхность детали, вызывая ее дополнительное упрочнение. В качестве дополнительных ударных элементов может выступать так же ворс самой щетки [28], авторами предложен метод комбинирования ворса проволочного инструмента из проволоки различной жесткости, для обеспечения дополнительного ударного воздействия на обрабатываемую поверхность.

С целью решения проблемы с размещением ЭМП при обработке внутренних поверхностей деталей авторами [53] предложено выполнять металлическую щетку с брусками из материала покрытия и узлами их прижатия, располагающимися между ворсом щетки, причем данные узлы закреплены со стороны основания, а элементы материала покрытия обращены наружу ворса. Что позволяет обрабатывать внутренние поверхности.

Для обработки сложных наружных и внутренних поверхностей предложено использование секционных щеток [54, 55]. Применяя блоки из отдельных секций появляется возможность обработки эвольвентных поверхностей зубчатых колес, шлицевых поверхностей и других сложных по конфигурации, в том числе и внутренних поверхностей. Для интенсификации процесса упрочнения при плакировании гибким инструментом дисковые щетки также изготавливают секционными [56]. Секции у данного вида щеток расположены по окружности, и зачастую, секции чередуются: по жесткости ворса, либо с пустыми секциями без ворса. Что увеличивает энергию удара ворсинок о поверхность обрабатываемого изделия.

Для повышения производительности процесса плакирования и снижения экономических затрат на проволочные щетки были разработаны конструкции гибкого инструмента [29, 57], обеспечивающие его переналадку по мере износа (или полного выхода из строя) ворса щетки. Конструкция механической щетки [57] отличается тем, что каждая секция в цилиндрической оправе закреплена затяжным винтом с возможностью перемещения в радиальном направлении, что позволяет подстраивать длину ворса под необходимый размер.

\section{ЗАКЛЮЧЕНИЕ. НАПРАВЛЕНИЯ ДАЛЬНЕЙШЕГО РАЗВИТИЯ}

\begin{abstract}
Проанализированные работы показывают достаточную изученность процесса деформационного плакирования в плане множества возможных схем реализации процесса, возможности интенсификации процесса дополнительными физическими воздействиями, проработанных конструкций гибкого инструмента, элемента из материала покрытия и конструкций его подачи в зону обработки. Что позволяет использовать данный метод для различного сочетания условий процесса, различными материалами детали, инструмента и материала покрытия, с постоянно высокими показателями качества получаемых изделий.
\end{abstract}

Основное развитие метода происходит в направлении усовершенствования работы схемы обработки, предложенной изобретателем Абиндером А.А. (рис. 2б). Большое количество различных методик обработки предложено изобретателями, которые охватывают практически все различные сферы применения метода плакирования и позволяют наносить покрытия различного функционального назначения на поверхности изделий машиностроения.

Дальнейшие тенденции развития данного метода, скорее всего, будут направлены на автоматизацию процесса. Так как для нормальной работы проволочного инструмента в режиме нанесения покрытия на обрабатываемую поверхность детали необходимо постоянство основных параметров, характеризующих этот процесс (скорости вращения ворса гибкого инструмента, усилия прижатия механической щетки к обрабатываемому изделию, температуры процесса и д.р.).

Автоматизация процесса предусматривает управление основными параметрами, следовательно, необходимы зависимости этих параметров от входных параметров процесса плакирования. Что ставит пред нами задачу детального изучения математических моделей описывающих данный процесс. 


\section{Impact Factor:}

\begin{tabular}{ll|lr} 
ISRA $($ India & $=\mathbf{1 . 3 4 4}$ & SIS $($ USA) & $=\mathbf{0 . 9 1 2}$ \\
ISI $($ Dubai, UAE) $=\mathbf{0 . 8 2 9}$ & PИНЦ $($ Russia $)=\mathbf{0 . 1 7 9}$ \\
GIF $($ Australia) $=\mathbf{0 . 3 5 6}$ & ESJI $($ KZ) & $=\mathbf{1 . 0 4 2}$ \\
JIF & $=\mathbf{1 . 5 0 0}$ & SJIF $($ Morocco $)=\mathbf{2 . 0 3 1}$
\end{tabular}

Исследование выполнено под руководством Гуляева В.А., к.т.н, доцент и Зотова А.B., cm. преп. кафедры «Оборудование $и$ технологии машиностроительного производства» ФГОБУ ВПО «Тольяттинский государственный университет», Россия

\section{References:}

1. Ancupov VP (1999) Teorija i praktika plakirovanija izdelij gibkim instrumentom. Magnitogorsk: MGTU im. G.I. Nosova, 1999. $241 \mathrm{p}$.

2. (1961) A.s. 139892 USSR, MKI S 23 S 17/00. Avtomat dlja serebrenija ciferblatov chasov metodom natiranija / I.M. Smirnova, N.A. Nikolaev, S.D. Krylov // Otkrytija. Izobretenija. 1961. № 14. pp. 59.

3. Bokov AI (2001) Povyshenie dolgovechnosti detalej metallurgicheskogo oborudovanija metodom plakirovanija gibkim instrumentom $\mathrm{s}$ uchetom ego iznosa i ustalostnogo razrushenija: Dis. kand. tehn. nauk. Magnitogorsk, 2001. 126 p.

4. (1940) A.s. 57162 USSR, MKI S 23 S 17/00. Sposob nanesenija metallicheskih pokrytij / A.A. Abinder // Otkrytija. Izobretenija. 1940. № 6. pp. 1-3.

5. Onshin NV (2001) Razrabotka i issledovanie metoda drobnogo plakirovanija gibkim instrumentom dlja proizvodstva bimetallicheskih detalej metallurgicheskogo oborudovanija povyshennoj iznosostojkosti: Dis. kand. tehn. nauk. Magnitogorsk, 2001. 119 p.

6. (1995) Sovershenstvovanie tehnologicheskih processov na metallurgicheskom kombinate / A.A. Gosteev, E.G. Kozodaev, I.G. Gun, V.M. Salganik, V.P. Ancupov, I.Ju. Mezin, V.E. Hrebto, M.V. Chukin. - M.: Metallurgija, 1995. $-170 \mathrm{p}$.

7. (1992) A.s. 1733506 USSR, MKI S 23 S 26/00. Sposob nanesenija pokrytij / Ju.V. Sankin, V.D. Gusev // B.I. - 1992. № 18. pp. 101.

8. (1991) A.s. 1687646 USSR, MKI S 23 S 26/00. Sposob nanesenija pokrytij iz aljuminija i ego splavov / I.I. Osheverov, P.N. Smirnov, L.S. Belevskij, V.I. Kadoshnikov, A.Ja. Tonkonogov, V.V. Raevskij // B.I. - 1991. № 40. pp. 92.

9. (1991) A.s. 1682663 USSR, MKI F 16 S 33/12. Sposob poluchenija pary trenija skol'zhenija / V.P. Ancupov, L.S. Belevskij, I.I. Osheverov, P.N. Smirnov, V.A. Dosmanov, N.P. Zaharov, N.A. Pisarev, R.H. Tagirov // B.I. - 1991. № 37. pp. 144

10. (1991) A.s. 1671733 USSR, MKI S 23 S 26/00. Sposob nanesenija pokrytij na provoloku / I.I.
Osheverov, P.N. Smirnov, L.S. Belevskij, V.I. Kadoshnikov // B.I. - 1991. № 31. pp. 84.

11. (1991) A.s. 1668473 USSR, MKI S 23 S 26/00. Sposob nanesenija metallicheskogo pokrytija / V.I. Kadoshnikov, L.S. Belevskij, I.I. Osheverov, P.N. Smirnov, V.L. Trahtengerc // B.I. - 1991. № 29. pp. 121 .

12. (1990) A.s. 1558996 USSR, MKI S 23 S 26/00. Ustrojstvo dlja nanesenija metallicheskih pokrytij na lentu / I.I. Osheverov, P.N. Smirnov, A.A. Titova, N.P. Osheverova // B.I. - 1990. № 23. pp. 129.

13. (1986) A.s. 1258873 USSR, MKI S 23 S 8/60. Ustrojstvo dlja nanesenija pokrytij na provoloku / I.I. Osheverov, P.N. Smirnov, L.S. Belevskij, V.V. Krivoshhapov, V.I. Kadoshnikov // B.I. - 1986. № 35. pp. 109.

14. (1986) A.s. 1215923 USSR, MKI S 23 K 20/04. Sposob poluchenija bimetallicheskoj lenty / L.S. Belevskij, V.I. Kadoshnikov, I.I. Osheverov, P.N. Smirnov // B.I. - 1986. № 9. pp. 56.

15. Belevskij LS, Buhinik GV, Kadoshnikov VI (1987) Ustanovka dlja nanesenija pokrytija na provoloku i lentu mehanicheskim sposobom // Bjul. In-ta «Chermetinformacija». - 1987. №3. - pp.51-52.

16. Belevskij LS (1987) Poverhnostnoe plasticheskoe deformirovanie $\mathrm{s}$ odnovremennym naneseniem pokrytij // Izv. Vuzov. Chernaja metallurgija. - 1987. - №7. pp. 104-106.

17. (1990) A.s. 1586878 USSR, MKI S 23 K 26/00. Ustrojstvo dlja nanesenija metallicheskih pokrytij na lentu / P.N. Smirnov, I.I. Osheverov, L.S. Belevskij, A.A. Titova // B.I. 1990. № 31. pp. 76.

18. (1986) A.s. 1258875 USSR, MKI S 23 K 26/00. Sposob nanesenija pokrytij na provoloku / B.A. Nikiforov, I.I. Osheverov, P.N. Smirnov, L.S. Belevskij, V.V. Krivoshhapov, V.I. Kadoshnikov // B.I. - 1986. № 35. pp. 109.

19. Belevskij LS (1996) Plasticheskoe deformirovanie poverhnostnogo sloja i formirovanie pokrytija pri nanesenii gibkim instrumentom. - Magnitogorsk: Licej RAN, 1996. $-231 \mathrm{p}$.

20. Ancupov VP (1997) Tehnologicheskie osnovy poluchenija bimetallicheskih izdelij 
plakirovaniem gibkim instrumentom: Dis. dokt. tehn. nauk. Magnitogorsk, 1997. 323 p.

21. (1994) A.s. RU 2015853 C1, MPK B22F 7/00. Sposob poluchenija pokrytij na poverhnosti metallicheskih izdelij / Belevskij L.S., Haritonov A.O., Kutlubaev I.M., Serov N.V. // 1994.

22. (1999) A.s. RU 2138579 C1, MPK S23S 26/00. Sposob uprochnenija detalej s odnovremennym naneseniem kompozicionnyh pokrytij / Gromakovskij D.G., Bersudskij A.L., Kovshov A.G., Malyshev V.P., Ibatullin I.D. // 1999.

23. (2007) A.s. RU 2308542 C1, MPK S23S 26/00, 30/00. Sposob nanesenija metallicheskih pokrytij na rabochie poverhnosti detalej mashin / Bersudskij A.L., Malyshev V.P., Astrahanskij A.Ju. // 2007. №29.

24. (2010) A.s. RU 2399696 C1, MPK S23S 26/00. Sposob uprochnenija detalej s odnovremennym naneseniem pokrytija / Bersudskij A.L., Zhdanov A.G., Astrahanskij A.Ju., Malyshev V.P. // 2010. №26.

25. Bersudskij AL (1987) Ustojchivost' ostatochnyh naprjazhenij poverhnostnogo sloja pri trenii posle uprochnjajushhej obrabotki $\mathrm{s}$ odnovremennym naneseniem pokrytija: Mezhvuz. sb. nauchn. tr. Sverdlovsk: UPI, 1987. t. 11. pp. 24-27.

26. Bersudskij AL (1987) Mehanizm formirovanija antifrikcionnyh pokrytij pri uprochnjajushhej obrabotke: Mezhvuz. sb. nauchn. tr. Sverdlovsk: UPI, 1987. t. 11. pp. 24-27.

27. (1990) A.s. 1579744 USSR, MKI B 24 B 39/00. Ustrojstvo dlja nanesenija metallicheskih pokrytij na izdelija / V.S. Blinov, I.I. Osheverov, P.N. Smirnov, L.S. Belevskij // Otkrytija. Izobretenija. - 1990. № 27. pp. 6566.

28. (1988) A.s. 1433466 USSR, MKI A 46 B 7/10. Cilindricheskaja shhetka dlja obrabotki poverhnosti / V.S. Blinov, I.I. Osheverov, P.N. Smirnov, L.S. Belevskij, V.V. Belan // 1988. № 40.

29. (1992) A.s. 1705406 USSR, MKI S 23 S 26/00. Ustrojstvo dlja frikcionnogo mehanicheskogo nanesenija pokrytij / T.K. Soldatova // B.I. 1992. - № 2. - pp. 118.

30. (1992) A.s. 1784659 USSR, MKI S 23 S 26/00. Ustrojstvo dlja obrabotki poverhnostej / A.V. Titenok, V.V. Titenok // B.I. - 1992. - № 48. pp. 85.

31.(1993) Pat. 1793977 USSR, MKI V 21 V 28/02. Sposob uprochnenija metallicheskoj poverhnosti / L.S. Belevskij, A.I. Starikov, V.P. Ancupov i dr. // Otkrytija. Izobretenija. 1993. № 5. - pp. 188-189.

32. Kadoshnikov VI, Kadoshnikova ID (1987) Poverhnostnoe plasticheskoe deformirovanie $\mathrm{s}$ odnovremennym naneseniem pokrytij // Teorija mashin metallurgicheskogo i gornogo oborudovanija: Mezhvuz. sb. nauchn. tr. Sverdlovsk: UPI, 1987. Vyp. 11. pp. 24-27.

33. Belevskij LS, Zavalishhina EG (1990) Metallizacija poverhnosti stali metallicheskimi shhetkami // Teorija i praktika processov obrabotki kompozicionnyh i sploshnyh materialov: Mezhvuz. sb. nauchn. tr. Magnitogorsk: MGMI, 1990. pp. 88-92.

34. (1987) A.s. 1344588 USSR, MKI V 24 V 39/00. Ustrojstvo dlja nanesenija pokrytij na izdelie / S.S. Dudaev, P.N. Smirnov, I.I. Osheverov, L.S. Belevskij // Otkrytija. Izobretenija. 1987. № 38. pp. 72.

35. (1990) A.s. 1588803 USSR, MKI S 23 S 26/00. Ustrojstvo dlja nanesenija pokrytija na poverhnost' / S.P. Gurov, S.L. Goncharov, S.Ja. Klepak // Otkrytija. Izobretenija. 1990. № 32. pp. 114-115.

36. (1992) A.s. 1733502 USSR, MKI S 23 S 26/00. Ustrojstvo dlja nanesenija pokrytij / A.Ju. Happalaev, O.V. Cygulev, V.S. Veremchuk, B.A. Ljashenko, M.M. Abacharaev, A.V. Rutkovskij // Otkrytija. Izobretenija. 1992. № 18. pp. 100-101.

37. (1994) Konstrukcii slitkopodajushhih ustrojstv v processah nanesenija metallicheskih pokrytij / V.I. Kadoshnikov, V.S. Blinov, L.S. Belevskij, I.D. Kadoshnikova // Tez. dokl. Mezhgosudarstvennoj nauchn.-tehn. konf.: Sostojanie i perspektivy razvitija nauchnotehnicheskogo potenciala Juzhno-Ural'skogo regiona. Sekcii: Mashinostroenie, Gornoe delo. Magnitogorsk, 1994. pp. 44-45.

38. (1996) A.s. 1206068 USSR, MKI V 24 K 39/00. Sposob nanesenija pokrytija / L.S. Belevskij, V.I. Kadoshnikov, Ju.M. Mironov, I.D. Kadoshnikova // B.I. - 1996. № 3. pp. 54.

39. (1993) Pat. 2381077, MKI V 21 V 28/02. Sposob uprochnenija metallicheskoj poverhnosti / L.S. Belevskij, A.I. Starikov, V.P. Ancupov i dr. // Otkrytija. Izobretenija. 1993. № 5. - pp. 188-189.

40. (2007) A.s. RU 2224822 C1, MPK S23S 26/00, 30/00. Sposob nanesenija metallicheskih pokrytij na rabochie poverhnosti detalej mashin / Bersudskij A.L., Malyshev V.P., Astrahanskij A.Ju. // 2007. №29.

41.(1986) A.s. 1446194 USSR, MKI V 24 K 39/00. Sposob nanesenija pokrytija / L.S. Belevskij, V.I. Kadoshnikov, Ju.M. Mironov, I.D. Kadoshnikova // B.I. - 1986. № 3. p. 54.

42. (2010) A.s. RU 2384654 C1, MPK S23S 26/00. Sposob uprochnenija detalej s odnovremennym naneseniem pokrytija / Bersudskij A.L., Zhdanov A.G., Astrahanskij A.Ju., Malyshev V.P. // 2010. №26.

43. (1994) A.s. RU 2008367 C1, MPK B22F 7/00. Sposob poluchenija pokrytij na poverhnosti 
metallicheskih izdelij / Belevskij L.S., Haritonov A.O., Kutlubaev I.M., Serov N.V. // 1994.

44. (1990) A.s. GV 863087, MKI S 23 S 26/00. Ustrojstvo dlja nanesenija metallicheskih pokrytij na lentu / I.I. Osheverov, P.N. Smirnov, A.A. Titova, N.P. Osheverova // B.I. - 1990. № 23. pp. 129.

45. (1987) A.s. 15990354 SSSR, MKI V 24 V 39/00. Ustrojstvo dlja nanesenija pokrytij na izdelie / S.S. Dudaev, P.N. Smirnov, I.I. Osheverov, L.S. Belevskij // Otkrytija. Izobretenija. 1987. № 38. pp. 72.

46. (1999) A.s. RU 32719 C1, MPK S23S 26/00. Sposob uprochnenija detalej s odnovremennym naneseniem kompozicionnyh pokrytij / Gromakovskij D.G., Bersudskij A.L., Kovshov A.G., Malyshev V.P., Ibatullin I.D. // 1999.

47. (1992) A.s. 1573054 USSR, MKI S 23 S 26/00. Ustrojstvo dlja nanesenija pokrytij / A.Ju. Happalaev, O.V. Cygulev, V.S. Veremchuk, B.A. Ljashenko, M.M. Abacharaev, A.V. Rutkovskij // Otkrytija. Izobretenija. 1992. № 18. pp. 100-101.

48. (1986) A.s. 1482980 USSR, MKI V 24 K 39/00. Sposob nanesenija pokrytija / L.S. Belevskij, V.I. Kadoshnikov, Ju.M. Mironov, I.D. Kadoshnikova // B.I. - 1986. № 3. pp. 54.

49. (1999) A.s. RU 2197562 C1, MPK S23S 26/00. Sposob uprochnenija detalej s odnovremennym naneseniem kompozicionnyh pokrytij / Gromakovskij D.G., Bersudskij A.L., Kovshov A.G., Malyshev V.P., Ibatullin I.D. // 1999.

50. Perepichka EV (1989) Ochistnouprochnjajushhaja obrabotka izdelij shhetkami - M.: Mashinostroenie, 1989. - 136 p.

51. Ancupov VP, Savel'eva RN, Savel'ev VB Matematicheskoe modelirovanie teplovyh processov pri nanesenii stal'nyh pokrytij provolochnymi shhetkami // Izv. vuzov. Mashinostroenie. - 1994. - № 10-12. - pp. 115119.

52. Ancupov VP, Savel'ev VB, Kadchenko SI (1994) Matematicheskoe modelirovanie poverhnosti metalla pri poverhnostnom plasticheskom deformirovanii shhetkami // Izv. vuzov. Chernaja metallurgija. - 1994. - № 11. pp. 30-32.

53. (1986) A.s. 1659531 USSR, MKI V 21 K $23 / 22$. Sposoby proizvodstva bimetallicheskoj stalealjuminevoj provoloki / L.S. Belevskij, P.N. Smirnov, I.I. Osheverov, B.A. Nikiforov, G.V. Buhinik, V.E. Bazarova, V.I. Kadoshnikov // B.I. - 1986. № 45. pp. 34.

54. (1999) A.s. RU 2362664 C1, MPK S23S 26/00. Sposob uprochnenija detalej s odnovremennym naneseniem kompozicionnyh pokrytij / Gromakovskij D.G., Bersudskij A.L., Kovshov A.G., Malyshev V.P., Ibatullin I.D. // 1999.

55. (1994) A.s. RU 2053106 C1, MPK B22F 7/00. Sposob poluchenija pokrytij na poverhnosti metallicheskih izdelij / Belevskij L.S., Haritonov A.O., Kutlubaev I.M., Serov N.V. // 1994.

56. (2010) A.s. RU 2384654 C1, MPK S23S 26/00. Sposob uprochnenija detalej s odnovremennym naneseniem pokrytija / Bersudskij A.L., Zhdanov A.G., Astrahanskij A.Ju., Malyshev V.P. // 2010. №26.

57. (2007) A.s. RU 27999 C1, MPK S23S 26/00, 30/00. Sposob nanesenija metallicheskih pokrytij na rabochie poverhnosti detalej mashin / Bersudskij A.L., Malyshev V.P., Astrahanskij A.Ju. // 2007. №29. 